H I G H L I G H T S

IN BRIEF

TECHNOLOGY

Scanometric DNA detection with nanoparticle probes.

Taton, T. A. et al. Science 289, 1757-1760 (2000).

In microarray experiments, the DNA hybridizing to the chip is usually labelled with a fluorescent dye. These authors have labelled the DNA with a gold nanoparticle. The specificity of the hybridization reaction allows single-base mismatches to be detected and the sensitivity of detection is increased by two orders of magnitude relative to a fluorescent label. The method could have important applications such as single nucleotide polymorphism screening in the analysis of human disease.

\section{HUMAN GENETICS}

An SNP map of the human genome generated by reduced representation shotgun sequencing.

Altshuler, D. et al. Nature 407, 513-516 (2000).

\section{An SNP map of human chromosome 22.}

Mullikin, J. C. et al. Nature 407, 516-520 (2000).

Single nucleotide polymorphisms (SNPs) are the markers of choice for mapping complex disease loci. There is debate about how many will be needed, but these two papers report the first fruits of the SNP consortium - an academic-industrial collaboration aiming to identify around 300,000 SNPs covering the human genome. The papers present alternative approaches to SNP identification and suggest that the SNP consortium will easily exceed its goal.

\section{EVOLUTION}

\section{Mutators and sex in bacteria: Conflict between adaptive strategies.}

Tenaillon, O. et al. Proc. Natl Acad. Sci. USA 97, 10465-10470 (2000)

Mutator alleles, which increase the mutation rate, can spread in an asexual population because they remain associated with the favourable alleles that they generate. Although mutator strains of bacteria would be expected to reach a very high frequency during the fixation of advantageous alleles, this does not occur. Using simulation models, the authors show that even rare genetic exchanges, such as occur by transduction and conjugation, are sufficient to reduce the selective spread of mutator strains.

\section{YEAST GENETICS}

swi1 and swi3 perform imprinting, pausing and termination of DNA replication in S. pombe.

Dalgaard, J. Z. « Klar, A. J. S. Cell 102, 745-751 (2000).

In mating-type switching in S. pombe, DNA at the mat 1 locus is replaced by DNA from one of two silent mat loci by recombination. This occurs by the introduction of a chromosomal imprint at mat 1 during the $S$ phase - an event that is sensitive to the direction of DNA replication. This paper reports the roles of swil and swi3 in this process and shows that they pause DNA replication to allow the chromosomal imprint to be established and terminate replication to block the entry of a replication fork from the opposite direction.

\title{
Counting the calories to immortality
}

Restricing calorie intake in many organisms - from mammals to yeast - delays the ageing process and extends lifespan, but explaining why calorie restriction (CR) has this effect has eluded biologists for many years. Recent advances have implicated oxidative stress among the mediators of this phenomenon, but its molecular basis remains unknown.

Budding yeast are particularly suitable for studies into this mystery because their lifespan is measured by a finite number of cell divisions once completed, the cell dies - and because they are acutely sensitive to environmental nutrients. Yeast also accumulate ribosomal DNA (rDNA) circles with age - these are created by homologous recombination at the repetitive rDNA locus and are excised to form extrachromosomal circles that are toxic to the cell. By assaying these read-outs of ageing in mutant yeast, Lin and colleagues have now discovered a key mechanism that links CR to longevity genomic silencing.

To confirm the link between CR and longevity, the authors grew yeast on a low-glucose medium. This extended yeast lifespan, as did inactivating the genes that enable yeast to sense and respond to glucose. Next, the authors tested whether these glucose-insensitive mutants could rescue the shortened lifespan of the sir2 mutant. The sir2 gene encodes a NAD (nicotinamide adenine dinucleotide)-dependent histone deacetylase that modifies chromatin and silences gene expression at several genomic loci, including at repetitive rDNA. Loss of sir 2 causes a rapid accumulation of toxic rDNA circles, a phenotype that can be rescued by suppressing hyper-recombination at the rDNA locus. When hyper-recombination is suppressed, sir2 mutant cells return to a normal lifespan but do not further extend it when they are made to be glu- cose insensitive. These results gave the first indication that CR-mediated longevity requires Sir2.

So what's the connection between longevity, CR and Sir2? A possible molecular basis of CRinduced yeast longevity is the reduction of rDNA recombination and of rDNA circle formation - in longliving, glucose-insensitive yeast, for example, both recombination rates and rDNA circle formation are significantly reduced. The authors believe that this effect is mediated by increased genomic silencing by Sir 2 . Furthermore, if yeast NAD synthesis is disrupted then CR can no longer induce longevity. This, perhaps, is not surprising, as Sir2 activity is dependent on NAD, which is a key intermediate in energy metabolism. CR might therefore be having a twofold effect on ageing - by reducing oxidative stress, and by activating Sir2 through NAD to coordinate genomic silencing, stability and gene expression with the energy status of the cell. Sir2 has a mammalian homologue, so whether the long-life benefits of CR can be achieved without counting calories remains to be seen.

Jane Alfred

(2) References and links ORIGINAL RESEARCH PAPER Li, S.-J. et al Requirement of NAD and SIR2 for life-span extension by calorie restriction in Saccharomyces cerevisiae. Science 289, 2126-2128 (2000) FURTHER READING Campisi, J. Aging, chromatin and food restriction - connecting the dots. Science 289, 2062 (2000) WEB SITE Lenny Guarente's lab

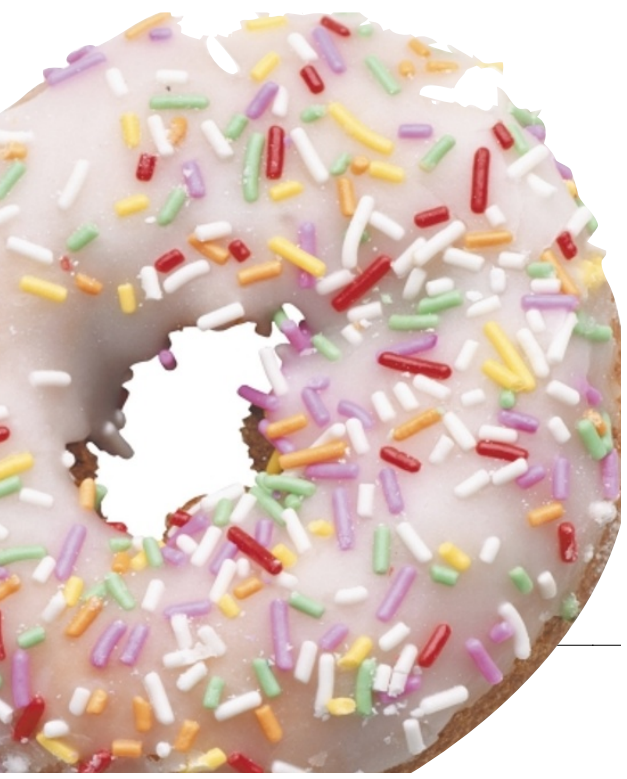

